\title{
The Neural Correlates of Recollection: Transient Versus Sustained fMRI Effects
}

\author{
Kaia L. Vilberg and Michael D. Rugg \\ Center for Vital Longevity and School of Behavioral and Brain Sciences, University of Texas at Dallas, Dallas, Texas 75235
}

Prior research has identified several regions where neural activity is enhanced when recollection of episodic information is successful. Here, we investigated whether these regions dissociate according to whether recollection-related activity is transient or sustained across the time that recollected information must be maintained before a behavioral judgment. Human subjects studied a series of word-picture pairs under the requirement to judge which of the denoted objects was smaller. Following each of 4 study sessions, a scanned test phase occurred in which a series of studied and unstudied words was presented. The requirement at test was to judge whether each word was old or new and, if judged old, to retrieve the associated study picture and hold it in mind until a cue appeared. The delay interval varied between two and eight seconds. The cue instructed subjects which of three different judgments should be applied to the retrieved picture. Separate responses were required when words were either deemed new or the associated image was not retrieved. Relative to studied words for which the associated picture could not be retrieved, words giving rise to successful recollection elicited transient responses in the hippocampus/parahippocampal cortex and retrosplenial cortex, and to sustained activity in prefrontal cortex, the intraparietal sulcus, the left angular gyrus and the inferior temporal gyrus. The finding that recollection-related activity in the angular gyrus tracked the period over which recollected information was maintained is consistent with the proposal that this region contributes to the online representation of recollected information.

\section{Introduction}

Relative to judgments of prior occurrence based on a sense of familiarity, the recollection of qualitative information about a past event leads to the engagement of a characteristic network of brain regions. These include the medial temporal lobe (MTL), retrosplenial and posterior cingulate cortices, and lateral parietal cortex in the vicinity of the angular gyrus, especially in the left hemisphere (Henson et al., 1999; Wheeler and Buckner, 2004; Yonelinas et al., 2005; Montaldi et al., 2006; Duarte et al., 2011; for review, see Spaniol et al., 2009; Kim, 2010). The consistency with which recollection effects have been identified in these regions, along with the finding that the same regions also demonstrate enhanced activity during successful recall, have led to the proposal that they constitute a "core recollection network" (Johnson and Rugg, 2007; Hayama et al., 2012).

The aim of the present study was to investigate the time courses of recollection-related activity in the different components of this network and thus to further understanding of their functional roles in recollection. The study was motivated by the prediction that in one component of the network at least-the left angular gyrus-recollection-related activity should covary with the time over which recollected information must be main-

\footnotetext{
Received June 28, 2012; revised Aug. 9, 2012; accepted Sept. 4, 2012.

Author contributions: K.L.V. and M.D.R. designed research; K.L.V. performed research; K.L.V. analyzed data;

K.L.V. and M.D.R. wrote the paper.

This research was supported by NIMH Grant 5R01MH072966.

Correspondence should be addressed to Kaia L. Vilberg, Center for Vital Longevity, University of Texas at Dallas, 1600 Viceroy Drive, Suite 800, Dallas, TX 75235. E-mail: kaia@utdallas.edu.

DOI:10.1523/JNEUROSCI.3065-12.2012

Copyright $\odot 2012$ the authors $\quad 0270-6474 / 12 / 3215679-09 \$ 15.00 / 0$
}

tained in service of a behavioral goal. The prediction derives from the proposal (Vilberg and Rugg, 2007, 2008; Yu et al., 2012) that the left angular gyrus contributes to the maintenance of recollected information, perhaps belonging to a network that supports the "episodic buffer" proposed by Baddeley (2000) to act as an interface between long-term memory and executive processes. This stands in contrast to an alternate proposal, whereby recollection-related activity in the region reflects its role in "detecting" the occurrence of recollection and reorienting attention to retrieved information (Cabeza et al., 2008; cf. Corbetta and Shulman, 2002; Cabeza et al., 2012). According to this account, angular gyrus activity should be transient, since the time required to reorient attention to recollected content will be independent of how long the content is subsequently maintained.

More generally, the present study allows the different components of the putative recollection network to be dissociated on the basis of the time courses of their recollection-related activity. Regions in which this activity is transient, and does not track the time over which recollected information is maintained, are candidates for enabling or initiating recollection but not for representing or maintaining recollected content. Rather, representation and maintenance of retrieved episodic information are likely supported by regions where activity is sustained across a maintenance interval.

To identify transient and sustained recollection-related responses we used functional magnetic resonance imaging (fMRI) to contrast neural activity elicited by a previously studied retrieval cue according to whether recollection of an associate of the cue succeeded or failed. Crucially, we varied the delay over which the recollected information had to be maintained before a decision 
contingent on the recollected information was made. Thus, we were able dissociate recollection-related activity according to whether it was unaffected by, or covaried with, the length of the delay.

\section{Materials and Methods}

Subjects. Twenty-one (10 female) native English speakers, aged $18-28$ years $($ mean $=21)$, took part in the experiment. Subjects reported themselves to be free of neurological disease and other contraindicated conditions for participation in an fMRI experiment. All subjects reported right-hand dominance. In accordance with the requirements of the University of Texas at Dallas Institutional Review Board, which approved the research, all subjects gave informed consent before participating. Imaging data from one subject were not collected due to a scanner error, and two subjects' data were excluded from analyses because of excessive motion ( $>5 \mathrm{~mm}$ within a test block). Thus, the results reported below use data from the 18 remaining subjects.

Experimental materials and procedure. The experiment consisted of 4 study-test cycles, with fMRI data acquired during each test block. Figure 1 shows a schematic of the structure of the study and test blocks. Study stimuli were word-picture pairs where each word and picture denoted a separate object. Pictures were presented in color on a gray background and all text was presented in black Helvetica font. Test items comprised single words. For each subject, 120 word-picture pairs were randomly assigned to serve as study items (30 pairs per block), and an additional 60 words were randomly assigned to be used as new test items (15 words per block). Items were selected from a pool of 403 picturename pairs previously used by Smith et al. (2004). During practice blocks, items were presented on a computer monitor. During study and test blocks in the scanner, items were presented on a screen positioned at the back of the scanner bore visible via a mirror attached to the head coil.

Before performing the first study block, subjects performed practice versions of the study and test tasks outside the scanner. The first practice session contained 4 study trials and 6 test trials. The second practice session contained 8 study and 12 test trials. Just before undertaking each task, verbal and written instructions were administered. The instructions were later repeated verbally just before each block of the experiment proper. To ensure compliance with task instructions, after completing each practice test, participants were questioned regarding their understanding of the task and were additionally asked to name the associated picture for at least one trial for which they indicated they recollected the paired picture (see below). Participants were given the opportunity to repeat either of the practice study-test lists if they were not yet comfortable with the task. Practice items were held constant across subjects. Responses during study and test blocks in the scanner were made using MRI-compatible button boxes.

At study, subjects viewed pairs of words and pictures presented on a gray background. On each trial, the word was presented to the left of a central fixation character $(+)$ and the picture to the right of the character. The task requirement was to imagine the two objects in real life and respond with the right index finger key if the item on the left was smaller than that on the right, the right middle finger key if the item on the right was smaller than that on the left, and the right ring finger key if the items were the same size. Each trial began with a $0.5 \mathrm{~s}$ central red fixation character, followed by the stimulus pair for $6 \mathrm{~s}$, followed by a centrally presented black fixation character for $0.5 \mathrm{~s}$. Participants were instructed to respond before the onset of the red fixation character signaling the beginning of the next trial. Each study block consisted of 30 trials.

For each test word, subjects were instructed to decide whether the word was old or new, and if old, to try to recollect and hold in mind the object that had been paired with the word at study until they were queried about one of three possible features of the recollected object. Following the presentation of the word, a cue appeared on the screen after a variable delay period. The cue indicated which of three questions the subject should answer regarding an attribute of the paired study picture, if it had been recollected. The three cues were 'shoebox', 'living', and 'household', representing the following questions, respectively: Would the recollected item fit inside a shoebox? Is the recollected item living or part of a living thing? Would the recollected item be found inside a house? By cueing these three different questions in an unpredictable sequence we aimed to prevent the adoption of a strategy whereby subjects classified recollected objects as soon as they were retrieved and then maintained the associated response over the delay period rather than a representation of the recollected object. On each trial, subjects were instructed to withhold their response until the appearance of the cue. If the test word was judged new, the new key under the left middle finger was to be pressed once the cue appeared. If the test word was judged old but the paired study picture could not be recollected, the 'old don't remember' key under the left index finger was to be pressed once the cue appeared. If the test word was judged old and the paired study picture was recollected, subjects were to press the key corresponding to a yes or no judgment to indicate their answer to the question posed by the cue with respect to the recollected object. The yes key was located under the right index finger and the no key was under the right middle finger. Response hand assignment was counterbalanced across subjects such that half of all participants used their left hand to answer the attribute question and the other half used their right hand.

Each test trial began with a central red fixation character for $0.5 \mathrm{~s}$, followed by a centrally presented test word for $1 \mathrm{~s}$, followed by a central black fixation for a variable delay $(2,4,6$, or $8 \mathrm{~s})$, a cue for $1 \mathrm{~s}$, a question mark for $2 \mathrm{~s}$, and a final black fixation character for a variable delay $(2,4$, or 6 s). Subjects were instructed to withhold responding until the appearance of the cue but to make a response before the offset of the question mark. A short break ( $<5 \mathrm{~min}$ ) was given between each study-test cycle. The ordering of the test trials was pseudo-randomized for each subject such that no more than 4 old or new trials occurred in succession. The durations of the black fixation character following word presentation at test were randomly assigned to old and new trials separately for each subject such that approximately equal proportions of each duration $(2,4$, 6 , and $8 \mathrm{~s}$ ) occurred within each of these trial types.

There are a number of assumptions underlying this test procedure. In keeping with similar prior studies (Vilberg and Rugg, 2007, 2009a,b), it is 
assumed that the contrast between the two classes of recognized items according to whether the studied associate was retrieved identifies neural activity selectively associated with recollection of the associate. It is further assumed that the contrast also identifies activity underlying the maintenance of recollected content across the delay period. Thus, not only is it assumed that when an associated object is recollected, subjects will comply with the instruction to maintain a representation of the object until the appearance of the response cue, it is also assumed that failure to retrieve the studied associate leads to the engagement of processes - such as iterative memory search and response preparation - that place lower demands on these maintenance operations.

MRI data acquisition. T1-weighted anatomical images $(240 \times 240$ matrix, $1 \mathrm{~mm}$ isotropic voxels) and blood oxygenation level-dependent (BOLD), $2^{\star}{ }^{\star}$-weighted echoplanar functional images (SENSE factor of 1.5 , flip angle $70^{\circ}, 80 \times 80$ matrix, $\mathrm{FOV}=24 \mathrm{~cm}, \mathrm{TR}=2000 \mathrm{~ms}$, TE $=$ $30 \mathrm{~ms}$ ) were acquired using a 3T Philips Achieva MRI (Philips Medical Systems) scanner equipped with a 32 channel receiver head coil. Three hundred and eleven volumes were acquired during each test block. Each volume comprised 33 slices oriented parallel to the AC-PC (anterior/ posterior commissure) line (thickness $3 \mathrm{~mm}, 1 \mathrm{~mm}$ interslice gap, $3 \mathrm{~mm}$ isotropic voxels) acquired in an ascending sequence. The first 5 volumes of each scanning session were discarded to allow equilibration of tissue magnetization.

Data analysis. Statistical Parametric Mapping (SPM8, Wellcome Department of Cognitive Neurology, London, UK), run under Matlab R2010a (MathWorks) was used for fMRI data analysis. Functional images were subjected to realignment (to the mean image), slice timing correction (using the middle slice as the reference), reorientation, spatial normalization to a standard echoplanar imaging template [based on the Montreal Neurological Institute (MNI) reference brain; Cocosco et al., 1997] and smoothing with an $8 \mathrm{~mm}$ full-width half-maximum Gaussian kernel. Each subject's structural volume was normalized to the MNI T1 template before averaging to create an across-subjects $(N=18)$ mean image. Functional analysis was performed using a General Linear Model (GLM) in which a delta function was used to model neural activity at item onset (item-related activity). Delay-related activity was modeled with a boxcar that onset concurrently with the fixation character following word presentation. The boxcar varied in length with the duration of the fixation character $(2,4,6$, or $8 \mathrm{~s}$ following the offset of the test item). The delta and boxcar functions were convolved with a canonical hemodynamic response function to model the predicted BOLD response (Friston et al., 1998). Four event types were modeled: associative hits (old trials for which the correct answer was given to the cue with respect to the study associate), item hits (old trials for which an 'old don't remember' response was given), correct rejections (new trials for which a new response was given), and events of no interest such as trials associated with incorrect responses, omitted responses, and multiple responses (these different trial types were collapsed into a single category because there were no, or very few, trials of one or more types for most subjects). The model thus contained 4 event types for each of the item- and delayrelated regressors, for a total of 8 event types in the model. The model also included as covariates six regressors representing motion-related variance (three for rigid-body translation and three for rotation), and regressors modeling the separate scan sessions and the across-scan mean. An AR(1) model was used to estimate and correct for nonsphericity of the error covariance (Friston et al., 2002). As described above, both the delay interval (between the item onset and cue onset) and the intertrial interval were varied across trials. The purpose of introducing this jitter across trials was to reduce the collinearity between the item and delay regressors in the GLM and thus increase estimation efficiency of the hemodynamic responses associated with each regressor.

The subject-specific item and delay parameter estimates for associative hits, item hits, and correct rejections were taken forward to a two-way repeated-measures ANOVA as implemented in SPM, using the factors of type of trial type and regressor (item versus delay). Unless otherwise noted, contrasts derived from the ANOVA model were thresholded at $p<0.001$, uncorrected, with a 26 voxel extent threshold. As estimated using Monte Carlo simulations implemented with the 3dClustSim function in AFNI, this cluster extent threshold gave rise to a corrected whole-
Table 1. Response proportions and RTs for old and new trials

\begin{tabular}{llllll}
\hline \multirow{2}{*}{ Response } & \multicolumn{3}{l}{ Proportions } & & RTs \\
\cline { 2 - 3 } \cline { 6 - 6 } \cline { 5 - 6 } & Old & New & & Old & New \\
\hline Yes/No correct & 0.31 & - & & $2491(361)$ & - \\
Yes/No incorrect & 0.21 & 0.02 & & $2607(487)$ & $2747(716)$ \\
Old don't remember & 0.33 & 0.12 & & $2283(589)$ & $2176(651)$ \\
New & 0.11 & 0.81 & & $2068(569)$ & $1925(467)$ \\
\hline
\end{tabular}

Response proportion columns do not sum to 1.00 due to exclusion of response omissions and trials upon which multiple key presses were made. RTs are displayed in milliseconds, with SDs in parentheses.

brain cluster-wise significance level of $p<0.05$. Exclusive masks were thresholded at $p<0.05$ one-tailed. Thresholds of $p<0.001$ were used in the inclusive masking of nonorthogonal contrasts. Coordinates of significant effects are reported in MNI space. Effects of interest are displayed on a section of the subjects' mean normalized structural image or rendered onto SPM's single subject rendering template.

In addition to the previously described GLM (hereafter referred to as the standard GLM), we created a second GLM in which a finite impulse response (FIR) model was used to estimate the time courses of the responses elicited by the three event types of interest (associative hits, item hits, and correct rejections) as well as events of no interest. The time courses were separately estimated for the $2,4,6$, and 8 s delay conditions across 20 time points ranging from the volume onsetting $2 \mathrm{~s}$ before item onset to $18 \mathrm{~s}$ post-item onset. The FIR model was used to characterize the BOLD time courses from the peak voxels of clusters identified by contrasts implemented in the standard GLM. The time courses of trials associated with the $2 \mathrm{~s}, 4 \mathrm{~s}, 6 \mathrm{~s}$, and $8 \mathrm{~s}$ delay intervals were truncated at the $6 \mathrm{~s}, 8 \mathrm{~s}, 10 \mathrm{~s}$, and $12 \mathrm{~s}$ poststimulus onset time points, respectively. These time points were selected on the basis of the predicted time courses of the BOLD responses associated with each interval, obtained by convolving a canonical hemodynamic response function with boxcar functions extending across each delay interval. Unless otherwise noted, plotted time courses display across-subject mean parameter values after averaging, within-subjects, across the delay intervals contributing to each timepoint. The SEs represent across-subject variability of the averaged parameter estimates.

In addition to using the FIR model to depict the hemodynamic time courses for associative hit, item hit and correct rejection trials, we also used the data from this model to evaluate whether activity in regions identified in the standard GLM covaried with delay interval, as would be expected of a region supporting the maintenance of information across the interval. A comparison of the predicted time courses (see above) of activity associated with the 2 and $8 \mathrm{~s}$ delays indicated that activity associated with the shorter delay should have returned to baseline by 12-14 s stimulus-onset, whereas that associated with the $8 \mathrm{~s}$ delay should be sustained for an additional $4 \mathrm{~s}$ or more. Thus, by 12-14 s following the onset of the test item, activity associated with the long and short delay interval trials should have diverged.

\section{Results}

\section{Behavioral results}

The mean old item hit rate was $83.8 \%$, against a mean correct rejection rate of $80.8 \%$. The proportions of old and new items attracting each class of response and their associated response times (RTs) with respect to cue onset are given in Table 1. As can be seen from the table, for items judged old, subjects were more likely to respond to the cue with 'yes' or 'no' than to respond 'don't remember'. The mean numbers of associative hit, item hit, and correct rejection trials were 43,35 , and 44 respectively, with ranges of $11-75,13-66$, and $21-59$, respectively. A repeatedmeasures ANOVA on test RTs (associative hits, item hits, and correct rejections) revealed a main effect of response type, $F_{(1.7,28.7)}=28.66, p<0.001$. Subsequent pairwise comparisons revealed that correct rejections were made significantly faster than associative hits, $F_{(1,17)}=57.74, p<0.001$, and item hits, 
$F_{(1,17)}=34.75, p<0.001$. Additionally, associative hits were associated with longer RTs than item hits, $F_{(1,17)}=5.46, p<$ 0.05 .

\section{fMRI results}

The principal fMRI analyses focused on the outcomes of contrasts that directly identified the neural correlates of recollection, namely those between associative hit and item hit trials (see Materials and Methods). We also report the outcome of the contrasts between item hit and correct rejection trials for regions demonstrating either a transient or a sustained recollection effect, although with one exception (see below), we had no pre-experimental predictions concerning the outcome of these contrasts.

Transient recollection effects were identified in a two-stage procedure. First, using the error term from the ANOVA model described in Materials and Methods, we identified regions demonstrating associative hit $>$ item hit effects for the item regressor. Then, we exclusively masked the outcome of this contrast with the associative hit $>$ item hit contrast for the delay regressor (see Materials and Methods). Thus, the outcome of this procedure yielded clusters where recollection effects were exclusively associated with the item regressor. Sustained recollection effects were identified by the outcome of the associative hit $>$ item hit contrast for the delay regressor alone. As is illustrated in Figure 2 (see also Table 2), regions demonstrating transient effects included the left parahippocampal/fusiform gyrus, extending into the hippocampus, and left retrosplenial cortex, extending into the precuneus. The figure also illustrates the time courses for these effects (note that although correct rejections were not included in the contrasts from which the results described above were derived, the time courses for correct rejection trials are included in Figs. 2 and 3 for illustrative purposes). Consistent with the impression given by Figure 2 , inclusive masking of these transient recollection effects with the item hit $>$ correct rejection contrast (thresholded here and below, at $p<0.001$ ) for the item regressor identified a significant difference between these event types in retrosplenial/posterior cingulate cortex (61 voxels) but not in the MTL.

Sustained effects were identified in the left inferior frontal gyrus, inferior temporal gyrus, medial frontal gyrus, bilateral striatum, posterior cingulate, and posterior parietal cortex extending from the intraparietal sulcus (IPS) to the angular gyrus. Figure 3 illustrates the loci of several of these sustained effects, and their time courses. Inclusive masking of these effects with the item hit $>$ correct rejection contrast for the delay regressor revealed significant differences between these event types in the medial superior frontal gyrus (598 voxels), left inferior frontal gyrus (two clusters: 44 and 41 voxels respectively), left inferior temporal gyrus (34 voxels), and left intraparietal sulcus (188 voxels).

For the reasons noted in the Introduction, the question of whether left parietal cortex in the vicinity of the angular gyrus would demonstrate transient or sustained recollection effects was of particular interest. To address this question, we extracted the mean parameter estimates from all voxels within two anatomi-
Table 2. Transient and sustained recollection effects

\begin{tabular}{|c|c|c|c|c|}
\hline Region & $B A$ & HM & Location & $\begin{array}{l}\text { Peak } Z \\
\text { (\# of voxels) }\end{array}$ \\
\hline \multicolumn{5}{|l|}{ Transient } \\
\hline $\begin{array}{l}\text { Parahippocampal gyrus/ } \\
\text { hippocampus }\end{array}$ & & L & $-27,-34,-20$ & $4.12(73)$ \\
\hline Retrosplenial cortex & $29 / 30$ & LR & $-12,-58,16$ & $4.52(159)$ \\
\hline \multicolumn{5}{|l|}{ Sustained } \\
\hline Inferior frontal gyrus & 45 & L & $-48,32,13$ & $5.68(2389)$ \\
\hline Inferior frontal gyrus & 45 & L & $-51,26,28$ & \\
\hline Superior frontal gyrus & 6 & $\mathrm{R}$ & $6,35,46$ & \\
\hline Caudate nucleus & & $L / R$ & $9,11,1$ & $4.52(432)$ \\
\hline Subthalamic nucleus & & $\mathrm{R}$ & $9,-7,-8$ & \\
\hline Insula & & $\mathrm{R}$ & $33,23,-5$ & \\
\hline Inferior temporal gyrus & $20 / 37$ & L & $-57,-46,-14$ & $6.26(248)$ \\
\hline Inferior temporal gyrus & $20 / 37$ & $\mathrm{R}$ & $63,-37,-20$ & $4.99(60)$ \\
\hline Intraparietal sulcus & $7 / 40$ & L & $-33,-55,46$ & $5.22(876)$ \\
\hline Intraparietal sulcus & 7 & L & $-39,-55,55$ & \\
\hline Angular gyrus & 39 & L & $-30,-73,46$ & \\
\hline Angular gyrus & 39 & $\mathrm{R}$ & $42,-55,43$ & 4.39 (354) \\
\hline Angular gyrus & 39 & $\mathrm{R}$ & $39,-70,34$ & \\
\hline Cerebellum & & $\mathrm{R}$ & $12,-82,-29$ & $4.30(139)$ \\
\hline Cerebellum & & $\mathrm{R}$ & $27,-67,-35$ & \\
\hline
\end{tabular}

$Z$-values refer to the peak of each activated cluster. Cluster sub-peak coordinates are also listed. BA, Brodmann area; $H M$, hemisphere; L, left; R, right.

cally defined regions of interest (ROIs) that encompassed the anterior (PGa) and posterior (PGp) aspects of the left angular gyrus (Fig. 4). The ROIs were generated using maximum probability maps defined by the Anatomy toolbox v1.8 (Eickhoff et al., $2005,2006,2007)$. For each subject the data were extracted for the different delay conditions and trial types using the FIR model described previously (see Materials and Methods). As is illustrated in Figure 4 (top), both regions of the angular gyrus appear to demonstrate sustained recollection effects. To assess whether these effects were statistically significant, we subjected the time course data (see Materials and Methods) to ANOVA. For each region, the ANOVA had factors of condition (associative vs item 


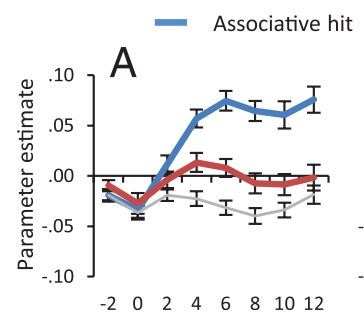

$\begin{array}{lllllllll}-2 & 0 & 2 & 4 & 6 & 8 & 10 & 12\end{array}$

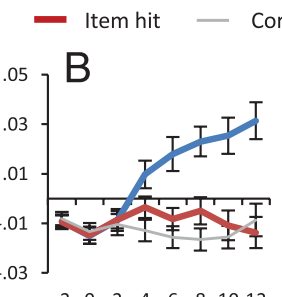

Correct rejection
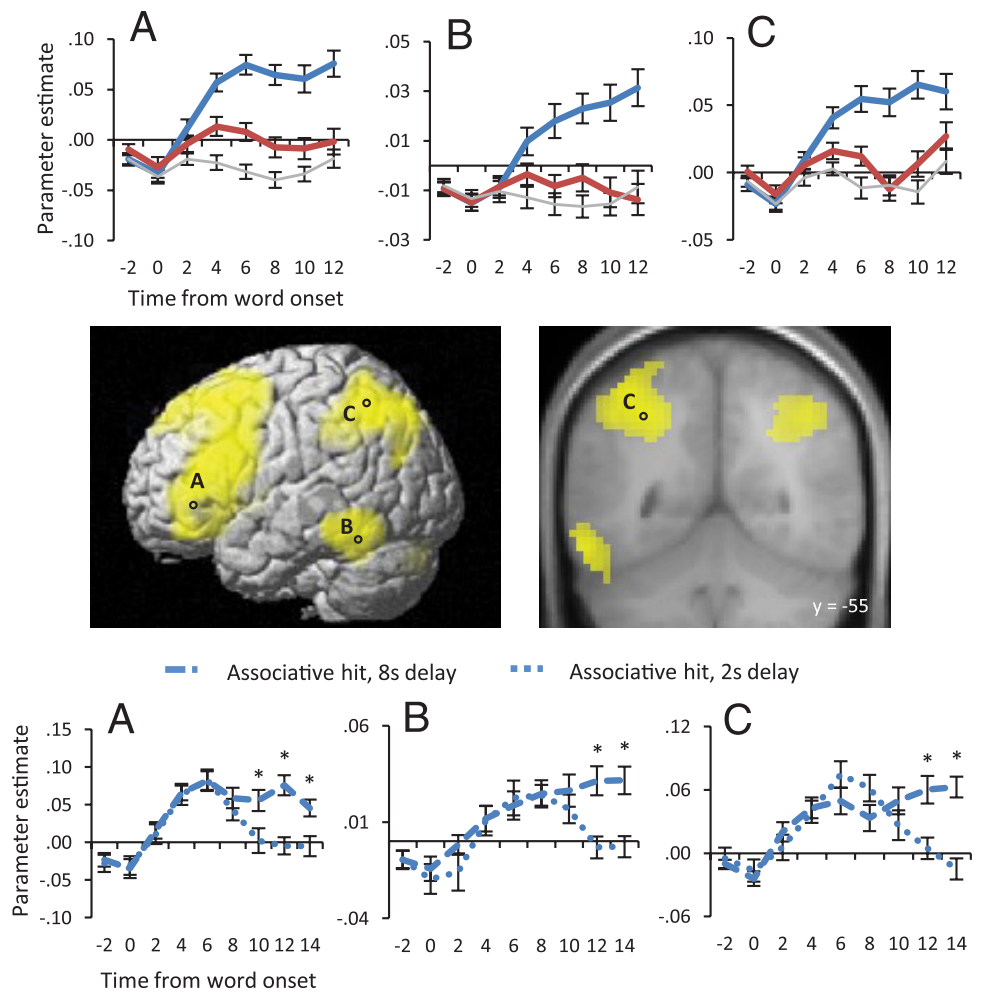

Figure 3. Top, Parameter estimates from the peak voxels of sustained effects in the left prefrontal, left inferior temporal, and left superior parietal cortex are shown across time from 2 s before word onset. The coordinates of the peak voxels shown are -48 , $32,13(\boldsymbol{A}) ;-57,-46,-14(\boldsymbol{B})$; and $-33,-55,46(\boldsymbol{C})$. Middle, Locations of the plotted recollection effects. The effects are projected onto SPM's single subject-rendered brain and are also displayed on a section of the mean across-subjects anatomical image. Bottom, Parameter estimates from the peak voxel of each effect are displayed separately for the 2 and $8 \mathrm{~s}$ delay interval associative hit trials. Asterisks indicate time points at which the two conditions differ at $p<0.01$.

hits) and time point (0-12 s post-word onset). Analysis of each ROI revealed main effects of condition and time point, $F_{(1,17)}=$ $25.62, p<0.001$ and $F_{(2.8,47.1)}=34.00, p<0.001$ respectively for PGa and $F_{(1,17)}=10.79, p<0.005$ and $F_{(3.0,50.6)}=7.22, p<0.001$ respectively for $\mathrm{PG}$. In addition, in each case the ANOVA revealed a condition by time point interaction, $F_{(2.5,42.3)}=6.12, p<$ 0.005 (anterior) and $F_{(2.4,40.2)}=3.32, p<0.05$ (posterior). Follow-up pairwise contrasts revealed that in $\mathrm{PGa}$, associative hits elicited greater activity than item hits by $4 \mathrm{~s}$ post-word onset, $F_{(1,17)}=16.81, p<0.005$, and continued to remain associated with significantly greater activity throughout the remainder of the analyzed time points, each $F_{(1,17)}>16$ and $p<0.005$. The same pattern was observed in PGp, where activity also differentiated between associative and item hits by $4 \mathrm{~s}$ following word onset, $F_{(1,17)}=9.49, p<0.01$, and the difference remained significant for each of the remaining time points, each $F_{(1,17)}>4$ and each $p<0.05$. Thus, both anterior and posterior aspects of the angular gyrus demonstrated recollection effects that were sustained across the maintenance interval. Subsidiary ANOVAs of the item hit and correct rejection time courses for these two ROIs revealed a main effect of time point in each case, $F_{(2.7,45.5)}=$ $14.81, p<0.001$ and $F_{(3.6,61.9)}=16.72, p<0.001$, for PGa and PGp, respectively. ANOVA of the PGa time courses additionally revealed a main effect of condition and a condition by time point interaction, $F_{(1,17)}=6.92, p<0.025$ and $F_{(3.3,56.6)}=3.60, p<$ 0.025 , respectively. Follow-up pairwise contrasts revealed that in $\mathrm{PGa}$, item hits elicited greater activity than correct rejections by 4 s post-word onset, $F_{(1,17)}=4.54, p<0.05$, and remained asso- ciated with greater activity throughout the remainder of the analyzed interval, each $F_{(1,17)}>7$ and $p<0.025$. Thus, PGa, but not PGp, demonstrated a sustained enhancement of activity for item hits relative to correct rejections.

Wewent on to assess whether recollectionrelated activity in the left angular gyrus was sensitive to the duration of the maintenance interval, as predicted if this activity is sensitive to the requirement to maintain recollected information across the interval (see Materials and Methods). For each angular gyrus region we performed an ANOVA on the averaged within-region parameter estimates for the associative hit trials derived from the FIR model. The ANOVA used the factors of maintenance interval ( $2 \mathrm{~s}$ vs $8 \mathrm{~s}$ ) and time point $(0-14 \mathrm{~s}$ after word onset). The bottom panel of Figure 4 illustrates the relevant time course data. ANOVA of the data from the PGa gave rise to main effects of maintenance interval, $F_{(1,17)}=7.69, p<0.025$, and time point, $F_{(3.1,52.4)}=14.98, p<0.001$, and to an interaction between these two factors, $F_{(3,50.9)}=5.91, p<0.005$. Planned pairwise contrasts confirmed that the parameter estimates for the 12 and $14 \mathrm{~s}$ time points were significantly greater for the $8 \mathrm{~s}$ than the $2 \mathrm{~s}$ trials $\left(t_{(17)}=3.31, p<0.005\right.$ one-tailed and $t_{(17)}=4.76, p<0.001$ one-tailed, respectively). The ANOVA of the data from the $\mathrm{PGp}$ region gave rise solely to a main effect of time point, $\left.F_{(2.7,46.2)}=3.28, p<0.05\right)$. Planned comparisons revealed that only in the case of the $14 \mathrm{~s}$ time point was activity greater for the $8 \mathrm{~s}$ than the $2 \mathrm{~s}$ delay interval $\left(t_{(17)}=\right.$ $1.86, p<0.05$ one-tailed; see Fig. 4 , bottom).

We repeated the foregoing analysis on the parameter estimates extracted from the peak voxels of the three principal regions where sustained effects were identified by the standard GLM analysis, namely, the left inferior frontal gyrus, left inferior temporal gyrus, and left IPS (Fig. 3, bottom). As in the case of the $\mathrm{PGa}$, each ANOVA revealed main effects of interval (for the left frontal, inferior temporal, and IPS peaks, $F_{(1,17)}=6.19,7.69$, and 4.69 , respectively, each $p<0.05$ ) and time point (for the left frontal, inferior temporal, and IPS peaks, $F_{(3.1,53.1)}=10.97$, $F_{(2.9,49.8)}=17.09$, and $F_{(4.3,72.7)}=13.64$, respectively, each $p<$ $0.001)$. An interaction between the two factors was also obtained for each region (frontal: $F_{(3.2,54.8)}=4.05, p<0.025$; temporal: $F_{(3.4,57.6)}=6.09, p<0.005$; and IPS: $\left.F_{(4,68.2)}=9.30, p<0.001\right)$. Follow-up contrasts revealed significantly greater associative hit activity at both the 12 and $14 \mathrm{~s}$ time points for the $8 \mathrm{~s}$ than the $2 \mathrm{~s}$ interval in each region (maximum $p<0.01$ ).

Finally, in light of prior reports of enhanced hippocampal activity for unstudied test items relative to studied items in studies of episodic memory retrieval (Daselaar et al., 2006), we addressed the question of whether MTL "novelty effects" could be identified in the present experiment. We searched for these effects by contrasting correct rejection and item hit trials, since prior studies have reported that novelty effects are greater for unrecollected than for recollected test items (Rugg and Yonelinas, 2003; Vilberg and Rugg, 2009c; Staresina et al., 2012). As we 
had an a priori reason for expecting such effects within the MTL, the cluster-wise correction was estimated for a volume that encompassed this region, rather than the whole brain. Thus, the contrasts were height thresholded at $p<0.001$ with a 7 voxel extent threshold (giving a corrected threshold of $p<0.05$ within the MTL). No MTL clusters could be identified that demonstrated transient novelty effects. By contrast, both the right amygdala and left anterior hippocampus showed sustained effects (amygdala: 21, 5, -23; 36 voxels; $Z=3.81$; hippocampus: -24 , $-10,-17 ; 22$ voxels; $Z=3.45)$. Figure 5 illustrates the effect in the left hippocampus along with the time courses for the peak voxel. The analogous contrasts using associative hits rather than item hits failed to identify either transient or sustained effects in the MTL.

\section{Discussion}

The present study investigated whether any neural correlates of successful recollection are sensitive to the requirement to maintain recollected information following its retrieval. By varying the interval over which recollected information had to be maintained, we were able to dissociate regions where the neural correlates of recollection were transient from regions where they were sustained over the maintenance interval. To the best of our knowledge, the present study is the first to characterize regional differences in the time courses of the neural correlates of recollection in this manner.

The regions identified here as sensitive to successful recollection include all of those held to be members of the putative core recollection network (Johnson and Rugg, 2007; Hayama et al., 2012; see also Kim, 2010). They include left inferior lateral parietal cortex (angular gyrus), retrosplenial/posterior cingulate cortex, and parahippocampal cortex/hippocampus. Additionally, we identified a recollection effect in left inferior temporal cortex. Whereas this region was not identified as a component of the core recollection network mentioned above, it has consistently been demonstrated to be recollectionsensitive in prior studies (Vilberg and Rugg, 2007; Kim, 2010). There was a striking dissociation among these regions with respect to their time courses: as is evident from Figures 2 and 3, whereas recollection effects in the retrosplenial cortex and medial temporal lobe were transient, the effects in the left angular gyrus and left inferior temporal cortex extended across the delay period.

The transient recollection response in the hippocampus is consistent with several related proposals concerning its role in



Item hit $\quad$ Correct rejection

Time from word onset

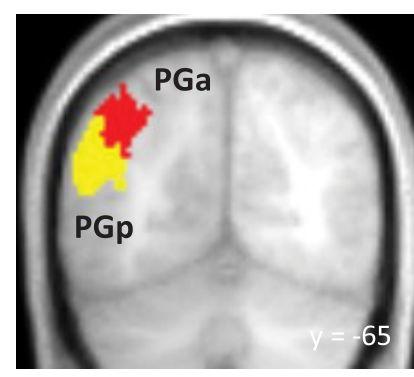

- Associative hit, 8s delay

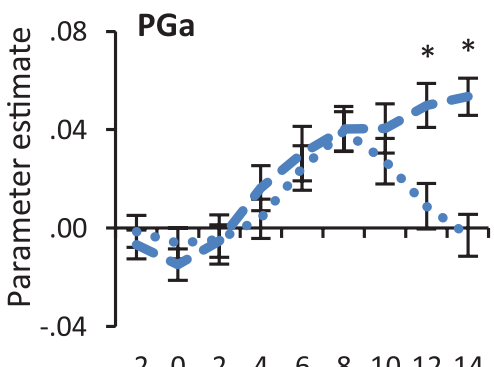

Time from word onset
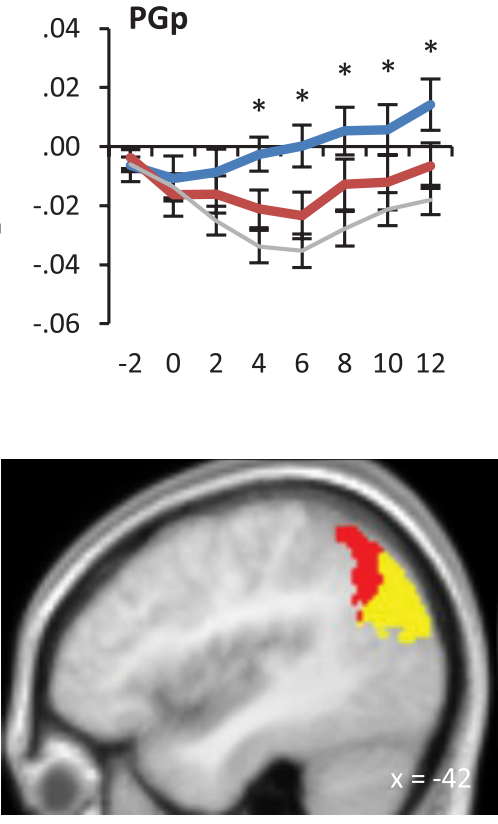

- Associative hit, 2s delay

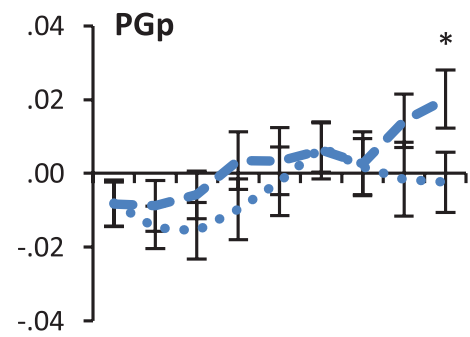

$\begin{array}{llllllllll}-2 & 0 & 2 & 4 & 6 & 8 & 10 & 12 & 14\end{array}$
Figure 4. Left anterior (PGa; red) and posterior (PGp; yellow) angular gyrus ROls are depicted on sections of the mean acrosssubjects anatomical image. Top, Parameter estimates averaged across all voxels within each ROl are shown from $2 \mathrm{~s}$ before word onset. Asterisks indicate time points at which associative hits and item hits differed: ${ }^{* *} p<0.005$ and ${ }^{*} p<0.05$. Bottom Parameter estimates are displayed separately for 2 and $8 \mathrm{~s}$ delay interval associative hit trials. Single asterisks indicate time points at which the two conditions differed at $p<0.05$.
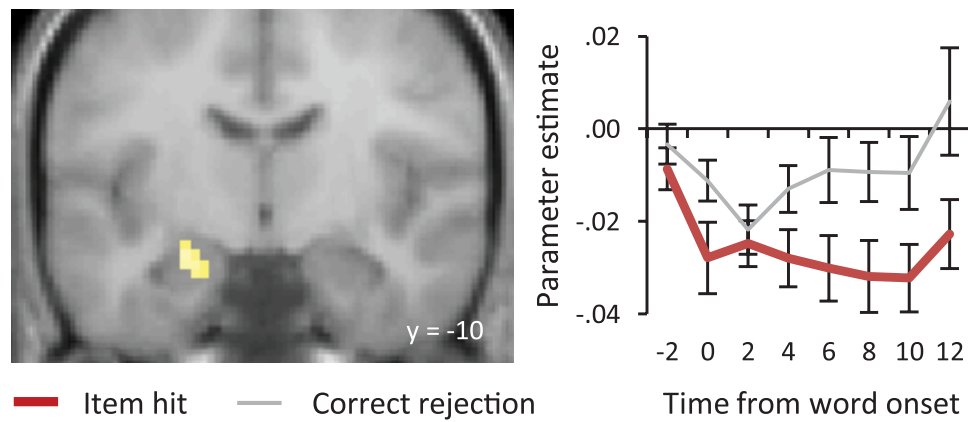

Time from word onset

Figure 5. Left, Sustained novelty effect in the left hippocampus $(-24,-10,-17)$. Right, Peak parameter estimates for correct rejections and item hits are displayed from $2 \mathrm{~s}$ before word onset. episodic memory (Alvarez and Squire, 1994; Rolls, 2000; Shastri, 2002; Norman and O'Reilly, 2003). According to these proposals, the hippocampus supports recollection of an event by reinstating the pattern of neural activity elicited when the event was initially experienced. Therefore, the hippocampus should play a 
key role in the initial establishment of a cortically mediated memory representation, but not necessarily its maintenance (see also Ranganath et al., 2005). It is less clear why retrosplenial and parahippocampal cortex also demonstrated transient responses. These regions are strongly interconnected with both the hippocampus and each other (Aggleton, 2012). According to Aggleton (2012) (see also Aggleton and Brown, 1999), retrosplenial cortex forms part of the "extended hippocampal" system, operating in concert with the hippocampus and diencephalon to support episodic memory. From this perspective, it may not be surprising that the two regions demonstrate similar time courses during successful retrieval. However, along with parahippocampal cortex (Diana et al., 2007; Eichenbaum et al., 2007), retrosplenial cortex has also been argued to play a role in context representation (Bar and Aminoff, 2003; Vann et al., 2009; Kveraga et al., 2011). Therefore, to the extent that recollection of the objects associated with studied test items involved retrieval of contextual information, these regions might have been expected to demonstrate sustained recollection effects, reflecting their contribution to the maintenance of recollected information. Whatever the functional significance of the retrosplenial and parahippocampal recollection effects identified here, the finding that the effects were transient suggests that they did not contribute to the representation of recollected information across the delay. One possibility is that the effects do indeed reflect contextual reinstatement (cf. Johnson and Rugg, 2007), but because the judgment following the delay period depended solely on the attributes of the retrieved object, only information about the object, rather than the object and its study context, was maintained.

A key question motivating the present study was whether sustained effects would be evident in the angular gyrus. This question is especially pertinent in light of the debate regarding whether the role of this region in episodic retrieval is better conceived of as mediating a transient, "bottom-up" attentional response (Cabeza et al., 2008, 2012), or as contributing to the online representation of recollected information (Vilberg and Rugg, 2008). The present findings are clearly incompatible with the first of these alternatives: according to both our whole-brain and ROI analyses, recollection-related activity in the angular gyrus was sustained across the delay period. As we have proposed previously (Vilberg and Rugg, 2008; see also Wagner et al., 2005), recollection effects in the angular gyrus may reflect its contribution to something like the episodic buffer proposed to act as an interface between episodic memory and executive functions (Baddeley, 2000). Whether this contribution would be to the storage of recollected information, however, or to attentional or control processes that operate on the stored content, is unclear. Alternatively, angular gyrus recollection effects might reflect the engagement of processes that operate not on recoded information residing in a buffer, but directly on retrieved information, perhaps integrating the different features of an episode into a coherent memory representation (cf. Shimamura, 2011; see also Cowan, 2008). The present findings do not permit adjudication between these or other hypotheses that are compatible with the finding of a sustained recollection effect in this region.

As already noted, the left inferior temporal gyrus has been reported to demonstrate recollection effects in several prior studies of episodic retrieval. The same region has also been implicated in the representation of conceptual information (Martin, 2007; Binder and Desai, 2011), especially the perceptual attributes of concrete concepts (Binder et al., 2005; Sabsevitz et al., 2005). We conjecture that the consistency with which the inferior temporal gyrus has been identified in previous studies of episodic retrieval reflects the popularity of concrete words and pictures of objects as experimental items, recollection of which would likely be associated with reinstatement of encoded conceptual information (Rugg et al., 2008). From this perspective, the present sustained inferior temporal recollection effects may reflect the contribution of this region to the representation and maintenance of attributes of the recollected object. Consistent with this suggestion, sustained effects in content-sensitive cortical regions have been reported in studies of visual working memory (Sala et al., 2003; Ranganath et al., 2004; but see Linden et al., 2012).

In addition to the regions implicated in previous studies of recollection, sustained effects were also evident in lateral PFC, bilateral superior parietal cortex and bilateral striatum. This "frontoparietal" network has frequently been reported to be active during the delay period of match-to-sample tasks (Mecklinger et al., 2002; Pessoa et al., 2002; Ranganath et al., 2004), and it has been proposed that the network supports control processes necessary to maintain representations of the sample item or items (Wager and Smith, 2003; Jonides et al., 2008). Given that the present task, like these working memory tasks, required the maintenance of task-relevant information across a delay, it is unsurprising that similar control processes were engaged. The precise extent of the overlap between patterns of activity associated with maintenance of perceptually and mnemonically derived information remains to be determined, however.

Relative to recognized items that failed to elicit recollection of their studied associate, new items elicited enhanced activity in the anterior MTL, including the hippocampus. Hippocampal novelty effects are thought to reflect encoding operations engaged by situationally novel stimulus events (Düzel et al., 2003; Stark and Okado, 2003; Nyberg, 2005; Lisman et al., 2011; Rugg et al., 2012). In contrast to the findings for hippocampal recollection effects, the present novelty effects were sustained across the maintenance interval. This finding, which is reminiscent of prior reports of greater sustained hippocampal activity for novel than for familiar items in delayed match to sample tasks (Ranganath and D'Esposito, 2001; Stern et al., 2001), raises the possibility that novelty-induced encoding can continue over relatively lengthy periods. If this interpretation is correct, the length of the delay period associated with new item trials should covary with the likelihood that the items are remembered on a later memory test. It will be of interest to see whether this prediction is fulfilled in future studies.

Although the principal focus of this study was the characterization of recollection effects, we also addressed the question whether any region demonstrating a recollection effect also demonstrated differences between item hits and correct rejections. We found one region where transient effects differed between these two classes of test item, and several regions where sustained effects differed. There are a number of potential explanations for these findings. For example, relative to correct rejections item hit trials were likely associated both with iterative retrieval attempts and the recovery of partial information about the study episode (noncriterial recollection). Elucidating the role of these or other processes will require studies in which they are brought under direct experimental control.

\section{Conclusion}

Despite the large literature describing the neural correlates of successful recollection, the present study is the first to investigate the temporal characteristics of recollection-related neural activ- 
ity. The findings demonstrate that recollection-sensitive brain regions can be dissociated according to the time courses of their respective recollection effects. In some regions the effects were relatively transient, while recollection-related activity in other regions covaried with the interval over which recollected information was maintained. Consistent with the proposal that the angular gyrus supports the online representation of recollected information but inconsistent with an attentional reorienting account, recollection effects in this region were sustained over the maintenance interval.

\section{References}

Aggleton JP (2012) Multiple anatomical systems embedded within the primate medial temporal lobe: Implications for hippocampal function. Neurosci Biobehav Rev 36:1579-1596.

Aggleton JP, Brown MW (1999) Episodic memory, amnesia, and the hippocampal-anterior thalamic axis. Behav Brain Sci 22:425-444.

Alvarez P, Squire LR (1994) Memory consolidation and the medial temporal lobe: a simple network model. Proc Natl Acad Sci U S A: 91: $7041-7045$.

Baddeley A (2000) The episodic buffer: A new component of working memory? Trends Cogn Sci 4:417-423.

Bar M, Aminoff E (2003) Cortical analysis of visual context. Neuron 38: $347-358$.

Binder JR, Desai RH (2011) The neurobiology of semantic memory. Trends Cogn Sci 15:527-536.

Binder JR, Westbury CF, McKiernan KA, Possing ET, Medler DA (2005) Distinct brain systems for processing concrete and abstract concepts. J Cogn Neurosci 17:905-917.

Cabeza R, Ciaramelli E, Olson IR, Moscovitch M (2008) The parietal cortex and episodic memory: an attentional account. Nat Rev Neurosci 9:613-625.

Cabeza R, Ciaramelli E, Moscovitch M (2012) Cognitive contributions of the ventral parietal cortex: an integrative theoretical account. Trends Cogn Sci 16:338-352.

Cocosco C, Kollokian V, Kwan RS, Evans A (1997) Brainweb: online interface to a 3D MRI simulated brain database. Neuroimage 5:S425.

Corbetta M, Shulman GL (2002) Control of goal-directed and stimulusdriven attention in the brain. Nat Rev Neurosci 3:201-215.

Cowan N (2008) What are the differences between long-term, short-term, and working memory? Prog Brain Res 169:323-338.

Daselaar SM, Fleck MS, Cabeza R (2006) Triple dissociation in the medial temporal lobes: recollection, familiarity, and novelty. J Neurophysiol 96: 1902-1911.

Diana RA, Yonelinas AP, Ranganath C (2007) Imaging recollection and familiarity in the medial temporal lobe: a three-component model. Trends Cogn Sci 11:379-386.

Duarte A, Henson RN, Graham KS (2011) Stimulus content and the neural correlates of source memory. Brain Res 1373:110-123.

Düzel E, Habib R, Rotte M, Guderian S, Tulving E, Heinze HJ (2003) Human hippocampal and parahippocampal activity during visual associative recognition memory for spatial and nonspatial stimulus configurations. J Neurosci 23:9439-9444

Eichenbaum H, Yonelinas AP, Ranganath C (2007) The medial temporal lobe and recognition memory. Annu Rev Neuroscience 30:123-152.

Eickhoff SB, Stephan KE, Mohlberg H, Grefkes C, Fink GR, Amunts K, Zilles K (2005) A new SPM toolbox for combining probabilistic cytoarchitectonic maps and functional imaging data. Neuroimage 25:1325-1335.

Eickhoff SB, Heim S, Zilles K, Amunts K (2006) Testing anatomically specified hypotheses in functional imaging using cytoarchitectonic maps. Neuroimage 32:570-582.

Eickhoff SB, Paus T, Caspers S, Grosbras MH, Evans AC, Zilles K, Amunts K (2007) Assignment of functional activations to probabilistic cytoarchitectonic areas revisited. Neuroimage 36:511-521.

Friston KJ, Fletcher P, Josephs O, Holmes A, Rugg MD, Turner R (1998) Event-related fMRI: characterizing differential responses. Neuroimage $7: 30-40$.

Friston KJ, Glaser DE, Henson RN, Kiebel S, Phillips C, Ashburner J (2002) Classical and Bayesian inference in neuroimaging: applications. Neuroimage 16:484-512.

Hayama HR, Vilberg KL, Rugg MD (2012) Overlap between the neural cor- relates of cued recall and source memory: evidence for a generic recollection network? J Cogn Neurosci 24:1127-1137.

Henson RN, Rugg MD, Shallice T, Josephs O, Dolan RJ (1999) Recollection and familiarity in recognition memory: an event-related functional magnetic resonance imaging study. J Neurosci 19:3962-3972.

Johnson JD, Rugg MD (2007) Recollection and the reinstatement of encoding-related cortical activity. Cereb Cortex 17:2507-2515.

Jonides J, Lewis RL, Nee DE, Lustig CA, Berman MG, Moore KS (2008) The mind and brain of short-term memory. Annu Rev Psychol 59:193-224.

Kim H (2010) Dissociating the roles of the default-mode, dorsal, and ventral networks in episodic memory retrieval. Neuroimage 50:1648-1657.

Kveraga K, Ghuman AS, Kassam KS, Aminoff EA, Hämäläinen MS, Chaumon M, Bar M (2011) Early onset of neural synchronization in the contextual associations network. Proc Natl Acad Sci U S A 108:3389-3394.

Linden DE, Oosterhof NN, Klein C, Downing PE (2012) Mapping brain activation and information during category-specific visual working memory. J Neurophysiol 107:628-639.

Lisman J, Grace AA, Duzel E (2011) A neoHebbian framework for episodic memory; role of dopamine-dependent late LTP. Trends Neurosci 34:536-547.

Martin A (2007) The representation of object concepts in the brain. Annu Rev Psychol 58:25-45.

Mecklinger A, Gruenewald C, Besson M, Magnié MN, Von Cramon DY (2002) Separable neuronal circuitries for manipulable and nonmanipulable objects in working memory. Cereb Cortex 12:1115-1123.

Montaldi D, Spencer TJ, Roberts N, Mayes AR (2006) The neural system that mediates familiarity memory. Hippocampus 16:504-520.

Norman KA, O’Reilly RC (2003) Modeling hippocampal and neocortical contributions to recognition memory: a complementary-learningsystems approach. Psychol Rev 110:611-646.

Pessoa L, Gutierrez E, Bandettini P, Ungerleider L (2002) Neural correlates of visual working memory: fMRI amplitude predicts task performance. Neuron 35:975-987.

Ranganath C, D'Esposito M (2001) Medial temporal lobe activity associated with active maintenance of novel information. Neuron 31:865-873.

Ranganath C, Cohen MX, Dam C, D'Esposito M (2004) Inferior temporal, prefrontal, and hippocampal contributions to visual working memory maintenance and associative memory retrieval. J Neurosci 24:3917-3925.

Ranganath C, Cohen MX, Brozinsky CJ (2005) Working memory maintenance contributes to long-term memory formation: neural and behavioral evidence. J Cogn Neurosci 17: 994-1010.

Nyberg L (2005) Any novelty in hippocampal formation and memory? Curr Opin Neurobiol 18:424-428.

Rolls ET (2000) Memory systems in the brain. Annu Rev Psychol 51:599_ 630.

Rugg MD, Yonelinas AP (2003) Human recognition: a cognitive neuroscience perspective. Trends Cogn Sci 7:313-319.

Rugg MD, Johnson JD, Park H, Uncapher MR (2008) Encoding-retrieval overlap in human episodic memory: a functional neuroimaging perspective. Prog Brain Res 169:339-352.

Rugg MD, Vilberg KL, Mattson JT, Yu SS, Johnson JD, Suzuki M (2012) Item memory, context memory and the hippocampus: fMRI evidence. Neuropsychologia. Advance online publication. Retrieved Sept. 5, 2012. doi: 10.1016/j.neuropsychologia.2012.06.004.

Sabsevitz DS, Medler DA, Seidenberg M, Binder JR (2005) Modulation of the semantic system by word imageability. Neuroimage 27:188-200.

Sala JB, Rama P, Courtney SM (2003) Functional topography of a distributed neural system for spatial and nonspatial information maintenance in working memory. Neuropsychologia 41:341-356.

Shastri L (2002) Episodic memory and cortico-hippocampal interactions. Trends Cogn Sci 6:162-168.

Shimamura AP (2011) Episodic retrieval and the cortical binding of relational activity. Cogn Affect Behav Neurosci 11:277-291.

Smith APR, Dolan RJ, Rugg MD (2004) Event-related potential correlates of the retrieval of emotional and nonemotional context. J Cogn Neurosci 16:760-775.

Spaniol J, Davidson PSR, Kim ASN, Han H, Moscovitch M, Grady CL (2009) Event-related fMRI studies of episodic encoding and retrieval: Metaanalyses using activation likelihood estimate. Neuropsychologia 47:17651779.

Staresina BP, Fell J, Do Lam ATA, Axmacher N, Henson RN (2012) Mem- 
ory signals are temporally dissociated in and across human hippocampus and perirhinal cortex. Nat Neurosci 15:1167-1173.

Stark CE, Okado Y (2003) Making memories without trying: medial temporal lobe activity associated with incidental memory formation during recognition. J Neurosci 23:6748-6753.

Stern CE, Sherman SJ, Kirchhoff BA, Hasselmo ME (2001) Medial temporal and prefrontal contributions to working memory tasks with novel and familiar stimuli. Hippocampus 11:337-346.

Vann SD, Aggleton JP, Maguire EA (2009) What does the retrosplenial cortex do? Nat Rev Neurosci 10:792-802.

Vilberg KL, Rugg MD (2007) Dissociation of the neural correlates of recognition memory according to familiarity, recollection, and amount of recollected information. Neuropsychologia 45:2216-2225.

Vilberg KL, Rugg MD (2008) Memory retrieval and the parietal cortex: a review of evidence from a dual-process perspective. Neuropsychologia 46:1787-1799.

Vilberg KL, Rugg MD (2009a) Left parietal cortex is modulated by amount of recollected verbal information. Neuroreport 20:1295-1299.
Vilberg KL, Rugg MD (2009b) Functional significance of retrieval-related activity in lateral parietal cortex: evidence from fMRI and ERPs. Hum Brain Mapp 30:1490-1501.

Vilberg KL, Rugg MD (2009c) An investigation of the effects of relative probability of old and new test items on the neural correlates of successful and unsuccessful source memory. Neuroimage 45:562-571.

Wager TD, Smith EE (2003) Neuroimaging studies of working memory: a meta-analysis. Cogn Affect Behav Neurosci 3:255-274.

Wagner AD, Shannon BJ, Kahn I, Buckner RL (2005) Parietal lobe contributions to episodic memory retrieval. Trends Cogn Sci 9:445-453.

Wheeler ME, Buckner RL (2004) Functional-anatomic correlates of remembering and knowing. Neuroimage 21:1337-1349.

Yonelinas AP, Otten LJ, Shaw KN, Rugg MD (2005) Separating the brain regions involved in recollection and familiarity in recognition memory. J Neurosci 25:3002-3008.

Yu SS, Johnson JD, Rugg MD (2012) Hippocampal activity during recognition memory co-varies with the accuracy and confidence of source memory judgments. Hippocampus 22:1429-1437. 